\title{
CATASTRO DIGITAL INTEGRADO: UN INSTRUMENTO DE DESARROLLO PARA LA CIUDAD DE TACNA
}

Responsable: Arq. Carlos Linares Torres Miembro: Arq. Yón Neira Arenas Miembro: Arq. Nilton Ferrel Zeballos

\section{RESUMEN}

El Planeamiento Urbano se materializa a través de la elaboración de Planes de Acondicionamiento Territorial, Planes de Desarrollo Urbano y Rural, Planes de Desarrollo de Asentamientos Humanos, etc. Estos deben ser el resultado de un análisis y diagnóstico situacional de los elementos físicos, sociales, económicos, legales, culturales y humanos que conforman un determinado territorio. Sin este conocimiento previo se corre el riesgo de modificaciones antes de los horizontes previstos o sencillamente su inaplicabilidad.

A partir del análisis de la situación del Catastro en la ciudad de Tacna y de los alcances y limitaciones de los Catastros elaborados por las instituciones vinculadas al tema, se procede a analizar específicamente el estado situacional en la Municipalidad Provincial de Tacna, determinar las situaciones negativas del servicio catastral y en qué términos las diferentes Áreas de la Municipalidad e instituciones vinculadas utilizan la información catastral.

A partir del análisis de la información recabada se procederá a la elaboración de las conclusiones, las cuales servirán para proponer una alternativa de solución al problema planteado.

\section{ABSTRACT}

The Urban Planning materializes through Plans's elaboration of Territorial Conditioning, Urban Plans and Rural Development, Development plans of Informal Housings, etc. They must be stemmed from an analysis and situational diagnosis of the physical, social, economic, legal and cultural factors, also human beings that conforms a determined territory. Modifications before the foreseen horizons or simply its inapplicability, take risk without this previous knowledge.

Starting from the Cadastre's analysis in Tacna and the goals and limitations of the elaborated Cadastres for the linked institutions to the theme, it proceeds to examine specifically the situational status in Municipalidad Provincial de Tacna to determine the cadastral service's negative situations and what terms the different the Municipality's areas and linked institutions use cadastral information.Starting from the information's analysis gotten conclusions will be elaborated; they would serve to propose an alternative to solve this problem.

\section{INTRODUCCIÓN}

El vocablo parece originarse en Roma en los primeros años de nuestra era, a partir del relevamiento de información gráfica en tablillas de bronce, madera o mármol, Esta información registrada para cada propietario "per cápita", dio origen al "capitum registrum" 0 registro de propietarios $y$, por extensión, al registro de unidades territoriales pasibles de tributar, de donde se deriva "capitastrum" raíz etimológica común en todas las lenguas latinas y germánicas de la palabra catastro.

\section{Denifición:}

a. Censo y padrón estadistico de las fincas rústicas y urbanas. (Real Academia).

b. Inventario de elementos físicos, sociales, económicos, legales, culturales y humanos que conforman un territorio. (Pablo Vicuña Pommiér-2003). c. Sistema integrado por bases de datos que reúnen información sobre el registro y la propiedad del suelo, sus características físicas, la zonificación, los sistemas de información geográfica, el sistema de transporte y datos ambientales, socioeconómicos y demográficos. (Diego Alfonso Erba-2006).

El antecedente más remoto del Catastro en el Perú fue cuando en 1856 se encargó a las municipalidades la formación de catastros y la reunión de toda clase de datos estadísticos.

Puede apreciarse que, tradicionalmente, el catastro en el Perú ha mantenido de manera independiente tres Subsistemas catastrales: el urbano, el rural y el minero. Tanto el catastro rural como el catastro minero han sido administrados por el Gobierno Central, a través del PETT el primero; y del Instituto Nacional de Concesiones y Catastro Minero (INACC), el segundo; 
debiéndose resaltar que ambas instituciones han desarrollado, el los últimos años, una importante labor utilizando tecnología de punta. En la actualidad el catastro urbano es competencia de las municipalidades del país; sin embargo, en los últimos años, la Comisión de Formalización de la Propiedad Informal-COFOPRI ha efectuado el saneamiento físico-legal de predios urbanos ubicados en Asentamientos Humanos, Pueblos Jóvenes, Centros Poblados, Programas de Vivienda y Urbanizaciones Populares, implementando una Base Gráfica en la que se han incorporado más de un millón de predios urbanos.

El problema principal es el catastro urbano municipal que se encuentra disgregado, regulado por una profusa y diversa normatividad sobre la materia, sin que exista un único ente regulador de la misma.Hasta la fecha, ha faltado el planeamiento de una solución técnico económica que permita integrar la denominada "realidad urbana formal" en un único sistema catastral nacional. Un primer intento lo constituye la Ley $\mathrm{N}^{\circ}$ 28294-Ley que crea el Sistema Nacional Integrado de Catastro y su vinculación con el Registro de Predios, y su respectivo Reglamento; sin embargo, aún no quedan claros los alcances de la mencionada integración.

El siguiente cuadro muestra las principales instituciones que generan información para las actividades catastrales y la cantidad de predios catastrados.

\begin{tabular}{|l|l|r|r|r|c|}
\hline AMBITO & INSTITUCION & $\begin{array}{c}\text { N DE } \\
\text { PREDIOS }\end{array}$ & CATASTRADOS & $\begin{array}{c}\text { CATASTRO } \\
\text { DE } \\
\text { PENDIENTES }\end{array}$ & $\begin{array}{c}\text { PORCENTAJE } \\
\text { CATASTRADO }\end{array}$ \\
\hline Urbano & $\begin{array}{l}\text { Municipalidad } \\
\text { es }\end{array}$ & 3600000 & 400000 & 3200000 & $11 \%$ \\
\hline & COFOPRI & 1900000 & 1000000 & 900000 & $52 \%$ \\
\hline Rural & PETI & 3000000 & 2000000 & 1000000 & $67 \%$ \\
\hline Minero & INAAC & 80000 & 80000 & 0 & $100 \%$ \\
\hline TOTAL & & 8580000 & 3480000 & 5100000 & $40 \%$ \\
\hline
\end{tabular}

Fuente: Conferencia de Javier Alfaro Díaz: Seminario Internacional El Catastro Urbano y la Valuación de Inmuebles. Arequipa, enero 2006.

\section{PLANTEAMIENTO DEL PROBLEMA}

La ciudad de Tacna está ubicada en la costa meridional del Perú, a 558 m.s.n.m. en el centro del Valle del Caplina. Su ubicación estratégica a 36 kilómetros de la frontera con Chile le otorga potencialidades importantes como centro comercial de alcance internacional, especialmente con Chile y Bolivia.

En el contexto regional se constituye en el centro político, administrativo, comercial y de servicios más importante del departamento de Tacna.

La Municipalidad Provincial de Tacna cuenta con un Catastro cuyo origen data de 1987 y fue producto de una restitución fotográfica. En 1993 se levantó información catastral a través de la aplicación de fichas catastrales, habiendo sido actualizadas parcialmente en 1998, 2000 y 2003. En el año 2004 se implementó una Base Gráfica Catastral pero tiene información parcial y no está actualizada.

Si bien las municipalidades son competentes para planificar la organización del espacio físico y uso del suelo a través, entre otros aspectos, de la elaboración y mantenimiento del catastro urbano y rural, el asunto ha adquirido dimensiones nacionales a través de la dación de la Ley que crea el Sistema Nacional Integrado de Catastro.

La única forma de alcanzar los fines planteados en la norma citada en acápite anterior es el trabajo con tecnología digital, tanto en el proceso de levantamiento catastral (restitución fotogramétrica, estación total, etc.), como en el procesamiento de datos a través de un Sistema de Información Geográfica (SIG).

En este contexto, la implementación de un catastro digital integrado de la ciudad de Tacna resulta de necesidad prioritaria y, dado que el tema es muy amplio, es preciso definir que la investigación se realizará respecto a los aspectos físicos de los predios urbanos ubicados en la jurisdicción del distrito de Tacna.

\section{ANTECEDENTES}

\section{Localización Orgánica:}

No existe en el Organigrama Estructural de la Municipalidad Provincial de Tacna un espacio específico para el Catastro. Tampoco se menciona en el Reglamento de Organización y Funciones vigente. Tan sólo la Subgerencia de Acondicionamiento Territorial, Catastro y Control Urbano cuenta entre muchas otras, con un Área de Catastro que cumple con las funciones de actualización y mantenimiento catastral.

\section{Área de Catastro:}

\section{a. Infraestructura y Logística:}

El Área se ubica en un ambiente que no está debidamente adecuado en cuanto a espacio y que se comparte con las Áreas de Planeamiento Urbano, Habilitaciones Urbanas, Subdivisión de Tierras, Zonificación yVias.

Se cuenta con un software de base de datos alfanumérico inoperativo y que inclusive no representa ser adecuado para el optimo funcionamiento del Catastro Urbano 
Municipal además que no existe software de última generación como los Sistemas de Información Geográfica.

\section{b. Recursos Humanos:}

Los recursos humanos asignados al Área no corresponden a las reales necesidades de la misma, no existiendo empleado y/o funcionario dentro del Cuadro de Asignación de Personal para cumplir con dichas funciones.

\section{c. Documentación e Información Existente:}

- Archivo Físico: Dado que la información catastral se ha estado llevando mecánicamente, se cuenta con un amplio archivo físico, consistente en planos en original y copias, expedientes, fichas, etc.

- Archivo Digital: Hasta octubre del 2004 se contaba con valiosa información, propia y de otras instituciones vinculadas; sin embargo, ésta se encontraba dispersa en los equipos de las diferentes áreas y subutilizada.

\section{d. Servicios Técnicos y/o Administrativos Brindados:}

El Texto Único de Procedimientos Administrativos (TUPA) tiene un listado de servicios que han sido derivados para diligenciamiento en el Área de Catastro, correspondientes básicamente a la atención de certificaciones:

- Certificado de Ubicación Dentro/Fuera del Área de Expansión Urbana (CUFAEU)

- Certificado de Numeración

- Certificado Catastral

- Visación de Planos

- Ficha Catastral

- Certificado de Alineamiento

- Nomenclatura

\section{ANÁLISIS}

\section{Respecto a la Zona y Población Servida:}

La ciudad de Tacna en los últimos 20 años ha sufrido un crecimiento significativo en extensión superficial y población que no han sido necesariamente el resultado de un proceso planificado de desarrollo urbano sino que corresponde a procesos informales de ocupación de áreas extraurbanas o de sectores urbanos centrales que se han ido saturando espacialmente.

Dichos sectores de la ciudad no se encuentran adecuadamente catastrados y en muchoscasos no cuentan con la debida inscripción registral de la propiedad.

\section{Respecto a la Gravedad de la Situación Actual:}

La problemática del Catastro Municipal no es circunstancial, corresponde más bien a una situación de tipo estructural y permanente desde hace más de diez años y que se ha expresado negativamente en la calidad de servicios brindados por la Municipalidad.

En dicho contexto, la Unidad Formuladora de Proyectos de la Municipalidad Provincial de Tacna encargó la elaboración del Perfil del Proyecto: "Fortalecimiento e Implementación del Sistema Catastral Urbano Municipal del Distrito de Tacna", en el marco del Sistema Nacional de Inversión Pública; el mismo que fue declarado viable con fecha 01 de agosto del 2005 e inscrito en el Banco de Proyectos del Sistema Nacional de Inversión Pública con Código SNIP $N^{\circ} 17304$, con un monto aprobado ascendente a S/. 958908,00.

\section{Respecto a la Información Catastral Actual:}

Considerando que el Área de Catastro cuenta con un registro de 16478 unidades catastrales y en la última actualización catastral se trabajaron 6309 , se tendria un faltante de 10 169 unidades por actualizar.

\begin{tabular}{|c|c|}
\hline \multicolumn{2}{|c|}{$\begin{array}{l}\text { Clasificación Actual de Unidades } \\
\text { Catastrales por Área Construida }\end{array}$} \\
\hline DESCRIPCIÓN & $\begin{array}{l}\text { ÁREA } \\
\text { CONSTRUIDA } \\
(\mathrm{m} 2)\end{array}$ \\
\hline CASA HABITACIÓN & $2^{\prime}, 045746,84$ \\
\hline $\begin{array}{l}\text { ACTIVIDAD COMERCIAL DE MENOR } \\
\text { ESCALA (AREA } 12 \text { M2) }\end{array}$ & 55401,84 \\
\hline PUESTO DE MERCADILLO <12M2 & 691,64 \\
\hline PUESTO DE MERCADO DE ABASTOS $<12 \mathrm{M} 2$ & 694,99 \\
\hline PUESTO COMERCIAL $<12 \mathrm{M} 2$ & 4381,96 \\
\hline COMERCIO & 416558,50 \\
\hline INDUSTRIA & 58937,09 \\
\hline ESTABLECIMIENTO FINANCIERO Y MONETARIO & 13053,24 \\
\hline SALUD & 32222,12 \\
\hline INSTITUCIONAL & 30522,61 \\
\hline EDUCACIÓN INICIAL ESTATAL. & 2942,99 \\
\hline EDUCACION PRIMARIA ESTATAL. & 14185,61 \\
\hline EDUCACIÓN PRIMARIA-SECUNDARIA PARTICULAR. & 9010,24 \\
\hline EDUCACIÓN SUPERIOR UNIVERSITARIA ESTATAL. & $37,421.90$ \\
\hline EDUCACIÓN SUPERIOR INSTITUTO PARTICULAR. & 390.3 \\
\hline EDUCACIÓN ESPECIAL & 146 \\
\hline CULTURAL & 42728,37 \\
\hline EN CONSTRUCCIÓN O DESOCUPADO & $50,421.28$ \\
\hline ACTIVIDAD COMERCIAL SIN USO & $190,267.67$ \\
\hline NO ESPECIFICADO & 956.73 \\
\hline
\end{tabular}

Fuente: Personal de Rentas - Informática de la MPT. 
En la actualidad, se puede señalar que se cuenta con una Base Gráfica Catastral que contiene lo siguiente:

- Nomenclatura de Vias

- Numeración Municipal

- Código de Sectores, Manzanas y Lotes Catastrales

- Nomenclatura de Manzanas y Lotes de Habilitaciones Urbanas Ingresadas

- Valores Arancelarios 2005

- Uso de Suelo

- Límite de Asociaciones, Pueblos Jóvenes, Asentamientos Humanos, Urbanizaciones, Sectores, Juntas Vecinales, Centros Poblados,

Zona Monumental, Distritos, Área de Expansión Urbana

- Perimétricos de Partidas Registrales

- Otros.

A partir de la implementación de la mencionada Base Gráfica Catastral fue posible determinar que en el distrito de Tacna existen aproximadamente 25,000 lotes urbanos distribuidos en los distintos sectores catastrales, de conformidad al cuadro que se adjunta:

Lotes y Manzanas por Sector Catastral

\begin{tabular}{|l|r|c|}
\hline SECTOR CATASTRAL & $\begin{array}{c}\text { N } \\
\text { LOTES }\end{array}$ & $\begin{array}{c}\text { N } \\
\text { MANZA } \text { DE } \\
\text { MAS }\end{array}$ \\
\hline SĖCTOR IV & 417 & 28 \\
\hline SECTOR V & 3589 & 228 \\
\hline SECTOR VI & 2333 & 159 \\
\hline SECTOR VII & 2578 & 105 \\
\hline SECTOR VIII & 925 & 74 \\
\hline SECTOR IX & 894 & 70 \\
\hline SECTOR X & 3030 & 179 \\
\hline SECTOR XI & 2507 & 153 \\
\hline SECTOR XII & 1886 & 126 \\
\hline SECTOR XIII & 128 & 7 \\
\hline SECTOR XIV & 3080 & 180 \\
\hline SECTOR XV & 1873 & 210 \\
\hline SIN UBICACIÓN DEFINIDA & 2385 & 91 \\
\hline TOTAL & 25625 & 1610 \\
\hline
\end{tabular}

Fuente: Oficina de Catastro de la MPT.

\section{Respecto a la Recaudación Fiscal:}

De lo indicado puede interpretarse que existiria un aproximado de 9200 lotes sin catastrar que, a razón de $160,00 \mathrm{~m} 2$ por lote mínimo y considerando el monto del Impuesto Predial Mínimo de S/. 19.80, implicaría una recaudación adicional anual de S/. 182160,00 , en el peor de los casos. Esto en el hipotético caso de que los 9200 lotes no catastrados cuenten con una sola unidad catastral y estuvieran vacíos, lo cual es muy poco probable.
Siendo así, se tienen 10260 predios que habrían tenido ampliaciones que, a razón de $20 \mathrm{~m} 2$ de área techada adicional minima e Impuesto Predial mínimo de S/. 19,91 implicaría un ingreso adicional anual de S/. 204 276,60; que sumado a los S/. 182 160,00 determinados líneas arriba, significarian una recaudación adicional por concepto de Impuesto Predial de S/. 386 436,60 , sólo en el primer año.

\section{Relación Municipalidad Provincial de Tacna}

\section{- Instituciones Involucradas:}

De hecho, se consiguieron notables avances en las relaciones interinstitucionales:

- La SUNARP mostró total predisposición para la suscripción de un convenio tendiente a unificar su Base Catastral con la de la Municipalidad, para evitar los inconvenientes propios de trabajar con distintas georeferenciaciones.

-.COFOPRI solicitó la suscripción de un convenio que le permitiera continuar sus labores de saneamiento físico legal en la provincia.

-.EI PETT dejó de realizar acciones de saneamiento fisico legal en terrenos que se ubican dentro del Área de Expansión Urbana, indicando a sus administrados que cualquier acción tendiente a modificar sus predios debe tramitarse por ante la Municipalidad.

- La EPS Tacna facilitó su Base Catastral actualizada y, como ya se dijo, es a partir de ésta, que se ha implementado la Base Gráfica Catastral con que cuenta actualmente la Municipalidad Provincial de Tacna.

\section{CONCLUSIONES}

1. La falta de liderazgo en el cumplimiento de su funciones, por parte de la Municipalidad Provincial de Tacna, ha devenido en que cada institución de la ciudad se provea de su propio Catastro, desvinculados unos de otros y con la generación de los problemas propios de georeferenciaciones diversas.

2. Las Municipalidades Distritales de Alto de la Alianza, Ciudad Nueva, Pocollay y Gregorio Albarracín cubren el $65 \%$ del área urbana de la ciudad y albergan el $68 \%$ del número de lotes urbanos estimados; por tanto es imposible un Catastro Digital Integrado de la ciudad de Tacna sin su participación.

3. La información catastral con que cuenta la Municipalidad Provincial de Tacna se encuentra desactualizada. Existen aproximadamente 9,200 
Lotes sin catastrar en le distrito de Tacna, impidiendo su acceso a mejores condiciones de desarrollo e incremento de la rentabilidad económica del área donde se ubican.

4. Se estima que unos 10260 predios ubicados en los distintos sectores del distrito de Tacna han tenido ampliaciones de por lo menos $20 \mathrm{~m} 2$ de área techada, lo que, sumado a los lotes no catastrados, implica una recaudación adicional únicamente por concepto de Impuesto Predial, de S/. 386436,60 , sólo en el primer año.

5. La Base Gráfica Catastral levantada por la EPS Tacna es, en la actualidad, la más próxima a la realidad física, a nivel de manzaneo y frentes de lotización.

6. La Base Catastral Municipal implementada en el año 2004 en la Municipalidad Provincial de Tacna constituye una herramienta fundamental en el ingreso de cualquier trámite solicitado por los administrados y una importante referencia de contrastación con cualquier trabajo de implementación, actualización y/o mantenimiento que se ejecute.

7. Debe procederse a la inmediata implementación del Catastro Digital Integrado de la ciudad de Tacna, actualizando la información actual existente e incorporando las unidades catastrales no consideradas. Asimismo, a partir de dicha implementación, todo procedimiento que tenga relación con la información catastral del distrito debe contar previamente con Informe Técnico del Área de Catastro.

\section{PROPUESTA: CATASTRO DIGITAL INTEGRADO DE LA CIUDAD DE TACNA COMOALTERNATIVADE SOLUCIÓN}

El esfuerzo, pues, de elaborar el Catastro Digital Integrado de la Ciudad de Tacna debe ser una sumatoria de esfuerzos coordinados convenientemente que permitan estructurar un Sistema Catastral donde todos confluyan y de donde todos se sirvan.

\section{- Operatividad}

\section{Fase 1: Implementación y Coordinación}

La fase comprende dos frentes: En el frente interno se debe proceder a compilar y procesar la información existente en los archivos tanto digitales como materiales que se encuentra dispersa en las diferentes áreas de la Municipalidad.

En el frente externo se debe coordinar con las diferentes instituciones vinculadas con el tema de
Catastro con la finalidad de definir puntos concordantes tendientes a manejar una Base Gráfica Catastral unificada; intercambiando información y consolidando un soporte gráfico y alfanumérico iniciales.

\section{Fase 2: Ejecución}

Etapa I: Levantamiento de Lotes y Edición de Planos de Trazado y Lotización

Etapa II: Levantamiento de Fábrica y Elaboración de SIG

Etapa III: Integración con Bases Catastrales Distritales e Instituciones Vinculadas

Fase 3: Mantenimiento y Actualización - Recursos Humanos Materiales y Costos

Recursos Humanos S/. 390600.00

Etapa I: Levantamiento de Lotes y Edición de Planos de Trazado y Lotización

\begin{tabular}{|c|c|c|c|c|c|c|}
\hline Cargo & \multicolumn{2}{|c|}{ Número } & Remuneración & Meses & Parcial & Total S/. \\
\hline Coordinador (C) & \multicolumn{2}{|c|}{01} & 2500,00 & 8 & 20000.00 & \\
\hline 8 & \multicolumn{2}{|c|}{19200,00} & & & & \\
\hline Auxiliar de & \multicolumn{2}{|c|}{06} & 800,00 & 8 & 38400,00 & \\
\hline \multicolumn{7}{|l|}{ Topografia (A) } \\
\hline Monitor (M) & \multicolumn{2}{|c|}{01} & 1500.00 & 8 & 12000.00 & \\
\hline Editor Cad (EC) & \multicolumn{2}{|l|}{02} & 1000.00 & 8 & 16000,00 & \\
\hline Editor Gis (EG) & \multicolumn{2}{|l|}{01} & 100000 & 8 & 8000,00 & \\
\hline Abogado (AB) & \multirow{2}{*}{\multicolumn{2}{|c|}{01}} & 1500.00 & 8 & 12000,00 & \\
\hline Noticador(N) & 01 & & 600,00 & 8 & 4800.00 & 73600,00 \\
\hline \multicolumn{7}{|c|}{ Etapa II: Levantamiento de Fábrica y elaboración de GI } \\
\hline Cargo & & Número & Remuneración & Meses & Parcial & Total \\
\hline Coordinador (C) & & 01 & 2500,00 & 10 & 25000.00 & \\
\hline Verificador (V) & & 03 & 1800,00 & 10 & 54000,00 & \\
\hline Topógrafo (T) & & 02 & 1200.00 & 10 & 24000.00 & \\
\hline Auxilar de Topografil & ia (A) & 06 & 800.00 & 10 & 48000,00 & \\
\hline Monitor (M) & & 01 & 1500.00 & 10 & 15000.00 & \\
\hline Editor Cad $(E C)$ & & 01 & 1000,00 & 10 & 10000.00 & \\
\hline Editor Gis (EG) & & 02 & 1000,00 & 10 & 20000.00 & \\
\hline Abogado (AB) & & 01 & 1500,00 & 10 & 15000,00 & \\
\hline Notificador (N) & & 01 & 600,00 & 10 & 6000,00 & 7100 \\
\hline
\end{tabular}

Recursos Materiales S/. 248000,00

EQUIPO

\begin{tabular}{|l|c|l|l|l|}
\hline Nombre & Número & Costo Unitario & Parcial & TOTAL SI. \\
\hline Computadora Pentium IV & 06 & 3000,00 & 1800,00 & \\
\hline Plotter & 01 & 35000,00 & 3500000 & \\
\hline Scanner & 01 & 15000,00 & 15000,00 & \\
\hline Cámara Digital & 01 & 1000,00 & 1000,00 & \\
\hline Estación Total, Accesorios & 02 & 45000,00 & 90000,00 & \\
\hline Intercomunicador Portátil & 10 & 1500,00 & 15000,00 & 174000,00 \\
\hline
\end{tabular}

SOFTWARE

\begin{tabular}{|l|c|l|l|l|}
\hline Licencia & Número & Costo Unitario & Parcial & TOTAL S/ \\
\hline Software de Catastro & 01 & 6000,00 & 6000,00 & \\
\hline Auto Cad 2000 & 06 & 3500,00 & 21000,00 & \\
\hline ArcGis & 06 & 5500,00 & 33000,00 & 60000,00 \\
\hline
\end{tabular}

OTROS

\begin{tabular}{|l|l|l|}
\hline Descripción & Parcial & Total S/. \\
\hline Útiles & 5000,00 & \\
\hline Infraestructura & 1000,00 & \\
\hline Combustible & 3500,00 & \\
\hline Difusión & 2500,00 & \\
\hline Otros gastos & 2000,00 & 14000,00 \\
\hline
\end{tabular}




\section{BIOGRAFÍA}

Manual de Planificación Urbana Local Direccion General de Desarrollo Urbano Lima, Perú, 2000.

Manual de Normas y Especificaciones Tecnicas de Catastro Instituto Catarastal de Lima 2001 Alfaro Diaz, Javier, El Carastro y el Planeamiento Urbano en el Perçu Instituto de Investigaciçon y
Capacitaciçon Municipal Lima, 2004

Municipalidad Provincial de Tacna, Area de Rentas, Area de Catastro 2004.

Historia del Catastro,

www.catastro.gub.uy/historia.htm

La Contribuciom Territorial Urbana y el

Catastro Urbano entre 1993 y 1935,

www.icc.es/pdf/jor-cart-cadas/RVallejo.pdf.

Francia - desde 1815,

www.seneca.uab.es/historia/hn070332.htm 